\title{
Intraoperative maximal urethral closing pressure measurement: a new technique of tape tension adjustment in transobturator sling surgery?
}

\author{
Myung Beum Kang, Hyeong Gon Kim, Sung Hyun Paick, Yong Soo Lho, Hyoung Keun Park
}

Department of Urology, School of Medicine, Konkuk University

\begin{abstract}
Purpose: Tape tension adjustment is an essential procedure in mid-urethral sling surgery. The goal of this study was to determine if intraoperative maximal urethral closing pressure (MUCP) elevation could be used as a reference value for adequate tape tension adjustment and predict transobturator (TOT) sling surgery outcome.

Materials and Methods: A prospective study was performed using MUCP measurements just before tape insertion and just after tension adjustment during surgery. Clinical data including preoperative urodynamic results were collected. The cure rate was determined by questionnaire. Patients were divided into two groups. The MUCP elevation group included patients with a MUCP elevation of more than $10 \mathrm{cmH}_{2} \mathrm{O}$ before tape insertion; the others were regarded as the non-elevation group. The cure rate and pre- and postoperative clinical variables were compared between the two groups. Results: A total of 48 patients had TOT surgery. The MUCP elevation group $(\mathrm{n}=19)$ and the non-elevation group $(\mathrm{n}=29)$ were similar with regard to patient characteristics and the preoperative parameters including age, mixed incontinence prevalence, Q-tip angle, peak flow rate, MUCP and the valsalva leak point pressure (VLPP). The mean follow-up period was nine months. The cure rate was significantly higher in the group with MUCP elevation than in the non-elevation group $(84 \%$ vs. $52 \%, p=0.02)$. There was no significant difference in the mean postoperative peak flow rate between the two groups and there was no retention episode.
\end{abstract}

Conclusions: MUCP elevation of more than $10 \mathrm{cmH}_{2} \mathrm{O}$ just after tape insertion was a prognostic factor.

Key words: urinary incontinence; suburethral slings; treatment outcome; urodynamics

Int Braz, J Urol. 2011; 37: 751-757

\section{INTRODUCTION}

In 1996 a new surgical technique called the tension-free vaginal tape procedure was introduced by Ulmsten for stress urinary incontinence (SUI) (1). This procedure has become popular for treating SUI. Many studies have reported on a long-term success rate of more than $80 \%$ using both objective and subjective assessments $(2,3)$. Although the success rate of the mid-urethral sling is high, there are also many failed procedures. Therefore, identifying the factors that are associated with persistent SUI after surgery is extremely important. Though controversial, the low valsalva leak point pressure (VLPP), low maxi- mal urethral closing pressure (MUCP), the presence of intrinsic sphincter deficiency (ISD), presence of mixed incontinence, and the presence of a high grade cystocele are thought to be important prognostic factors $(4,5)$.

However, among the studies on the prognostic factors, there is no study on the tape tension adjustment during surgery as a possible prognostic factor. Recently, the tape shortening technique using clips showed a $60-70 \%$ success rate in patients with recurrence after a sling operation $(6,7)$. These studies suggest the importance of tape tension in sling surgery. In addition, a tension adjustable sling system, such as the Remeex readjustable sling, was 
developed and widely used for repair of SUI (8). Therefore, providing the proper tension during surgery to render the patient continent, is now a focus of study.

Tape tension adjustment is essential for mid urethral sling surgery. However, there have been no methods developed to evaluate the degree of tape tension during surgery. In addition, there is no consensus on the appropriate amount of tension. The amount of tension adjustment has occasionally been determined by the subjective judgment of the surgeon.

We hypothesized that tape tension would influence urethral pressure, which could be measured as the MUCP level in urodynamic testing.

The goal of this study was to determine whether MUCP changes influence the surgical outcome. The MUCP changes were studied before and after placement of the tape and the outcomes were assessed as potential prognostic factors of TOT surgery.

\section{MATERIALS AND METHODS}

From October 2007 to November 2008, the MUCP was prospectively measured just before and after tape insertion in the operating room. A single surgeon performed all TOT operations. The preoperative protocol included a history and physical examination, urinalysis, urine culture, uroflowmetry, PVR urine measurement, Q-tip test, and multichannel urodynamic studies. Urethral hypermobility was defined as when the angle of Q tip test was more than 30 degrees. Written consent was obtained from all patients.

Patients were included in the study if they were female with SUI or MUI (mixed urinary incontinence) and older than age 18.Patients were excluded if they had any urinary tract infection, malignancy, or were pregnant

TOT procedures were performed as follows. The procedures were performed under spinal anesthesia. After incision and minimal dissection of the vaginal wall, a 6Fr two-channel flexible UPP catheter was inserted to measure the MUCP using a multichannel urodynamic study instrument (Medtronics Inc., Minneapolis, MI, USA). Then, after removal of the catheter, Transobturator tape (Monarc) needles were inserted through out-in technique. After fill- ing the bladder with $250 \mathrm{~mL}$ physiological saline, cystoscopy was performed with the needles still in place.

After cystoscopy examination, a Monarc needle was connected to tape and pulled outside of the previous insertion site. The tension of the tape was adjusted and the catheter was re-inserted to measure the MUCP again. All measurements were completed and the vaginal wound was closed. Operator was blinded to intraoperative MUCP results and did not adjust the tension of the tape after second MUCP measurement.

Patients were discharged from the hospital the morning following the procedure, and were followed up with at one, six, and 12 months and then every year thereafter. The follow-up evaluation included a clinical history, physical examination with a stress test, uroflowmetry, and post-void residual (PVR) measurement.

Cure of incontinence after the procedure was defined as the absence of subjective leakage after coughing, laughing, or other abdominal-straining circumstances, and as the absence of objective leakage on stress cough tests when the patients felt a normal voiding sensation and other term was defined according to recent report (9).

All other cases were considered to be failures. The patients were divided into two groups by the change in the MUCP, i.e. the MUCP elevation group was defined as patients in whom the MUCP was elevated more than $10 \mathrm{cmH}_{2} \mathrm{O}$ after insertion of the tape compared to before insertion of the tape; the non-elevation group was shown by no change or elevation less than $10 \mathrm{cmH}_{2} \mathrm{O}$. Cure rate in the MUCP elevation group was compared to the non-elevation group. In addition, the MUCP changes were compared in patients who had successful procedures and patients who had failed procedures to determine whether it was a possible predictive factor.

Statistical analysis was performed using the Student t-test for continuous data, as well as Fisher's exact test and the chi-square test. Variables that had a p-value less than 0.05 in the univariate analysis were included in the multivariate logistic model. A $5 \%$ level of significance was used for all statistical testing and all statistical tests were 2-sided. The statistical analyses were performed using SPSS ${ }^{\circledR}$ 11.0. 


\section{RESULTS}

\section{Patient demographics}

A total of 48 patients, between 32 and 77 years of age (mean 50.3), underwent mid urethral sling surgery with the TOT technique by a single surgeon. No difference in the clinical characteristics was observed between the MUCP elevation group $(n=19)$ and the non-elevation group. Age, prevalence of mixed urinary incontinence, urethral hypermobility, peak flow rate, preoperative MUCP and leak point pressure were not significantly different between the two groups (Table-1). ence in the group with a failed procedure (from $40 \pm$ $18 \mathrm{cmH}_{2} \mathrm{O}$ to $42 \pm 22 \mathrm{cmH}_{2} \mathrm{O}$ ) (Figure-2).

\section{Complications}

De novo urgency or urge incontinence was present in one patient in the group with the MUCP elevation group and in seven patients in the non-elevation group. The bladder irritation symptoms were more severe in the MUCP elevation group; however, this difference was not significant. The postoperative peak flow rate and PVR was not significantly different in comparisons between the two groups. No patients developed urinary retention (defined as

Table 1 - Preoperative clinical parameter between elevation group and non elevation group.

\begin{tabular}{|c|c|c|c|}
\hline & MUCP elevation group & Non elevation group & p-value \\
\hline No. of patients & 19 & 29 & \\
\hline Mean age (years) & $49.7 \pm 8.7$ & $50.7 \pm 9.9$ & 0.72 \\
\hline Prevalence of urgency (\%) & 32 & 46 & 0.06 \\
\hline Prevalence of urge incontinence (\%) & 31 & 35 & 0.56 \\
\hline Prevalence of urethral hypermobility (\%) & 69 & 52 & 0.06 \\
\hline Prevalence of detrusor overactivity (\%) & 11 & 14 & 0.15 \\
\hline Mean $\mathrm{MUCP}\left(\mathrm{cmH}_{2} \mathrm{O}\right)$ & $60 \pm 24$ & $66 \pm 33$ & 0.16 \\
\hline Mean VLPP $\left(\mathrm{cmH}_{2} \mathrm{O}\right)$ & $100 \pm 15$ & $95 \pm 25$ & 0.10 \\
\hline Mean peak flow rate $(\mathrm{ml} / \mathrm{sec})$ & 25 & 26 & 0.70 \\
\hline
\end{tabular}

MUCP: maximal urethral closing pressure; VLPP: valsalva leak point pressure

\section{MUCP changes and cure rate}

The overall cure rate was $65 \%(n=35)$ and the mean follow-up period was nine months (6-15 months). The MUCP was elevated significantly from $36 \pm 17 \mathrm{cmH}_{2} \mathrm{O}$ (before placement of the tape) to $42 \pm 18 \mathrm{cmH}_{2} \mathrm{O}$ (after placement of the tape) in all patients $(\mathrm{p}=0.04)$. The MUCP was significantly elevated in the MUCP elevation group (from $32 \pm 15$ $\mathrm{cmH}_{2} \mathrm{O}$ to $51 \pm 17 \mathrm{cmH}_{2} \mathrm{O}$ ) but not in the non-elevation group (from $40 \pm 18 \mathrm{cmH}_{2} \mathrm{O}$ to $35 \pm 16 \mathrm{cmH}_{2} \mathrm{O}$ ).

The overall cure rate was significantly higher in the MUCP elevation group compared to the nonelevation group ( $84 \%$ vs. $52 \%)(\mathrm{p}=0.02)($ Figure-1).

In the group that was cured, the MUCP increased from $34 \pm 17 \mathrm{cmH}_{2} \mathrm{O}$ to $41 \pm 16 \mathrm{cmH}_{2} \mathrm{O}$ after placement of the tape; however, there was no differ-
PVR greater than $100 \mathrm{~mL}$ or did not void) or vaginal erosion during the follow-up period (Table-2).

\section{DISCUSSION}

Since tension-free vaginal tape has been introduced and used widely, many clinical prognostic factors have been reported including ISD, low VLPP, presence of cystocele, and mixed incontinence $(4,5)$. However, there has been little attention focused on the tension of the tape during surgery and the prognostic significance of the tape tension on the surgical outcome.

Initially, many surgeons thought it was unnecessary to provide tension on the tape. This was because the mechanism of the mid-urethral sling was 
Table 2 - Postoperative clinical parameter between MUCP elevation group and non elevation group.

\begin{tabular}{lccc}
\hline & MUCP elevation group & Non elevation group & P value \\
\hline Success rate (\%) & 84 & 52 & 0.02 \\
Mean MUCP change (cmH ${ }_{2}$ ) & $19 \pm 16$ & $5 \pm 17$ & 0.03 \\
No. of patients with de novo urgency and/or urge incontinence & 1 & 7 & 0.17 \\
Mean peak flow rate (mL/sec) & $18.2 \pm 8.8$ & $29 \pm 25$ & 0.72 \\
Mean post void residual (mL) & $24 \pm 29$ & 0.6 & 0.46 \\
Episode of Urinary retention & 0 & 0 & 0 \\
Episode of vaginal erosion & &
\end{tabular}

MUCP: maximal urethral closing pressure

Figure 1 - Comparison of cure rate between MUCP elevation group and non elevation group.

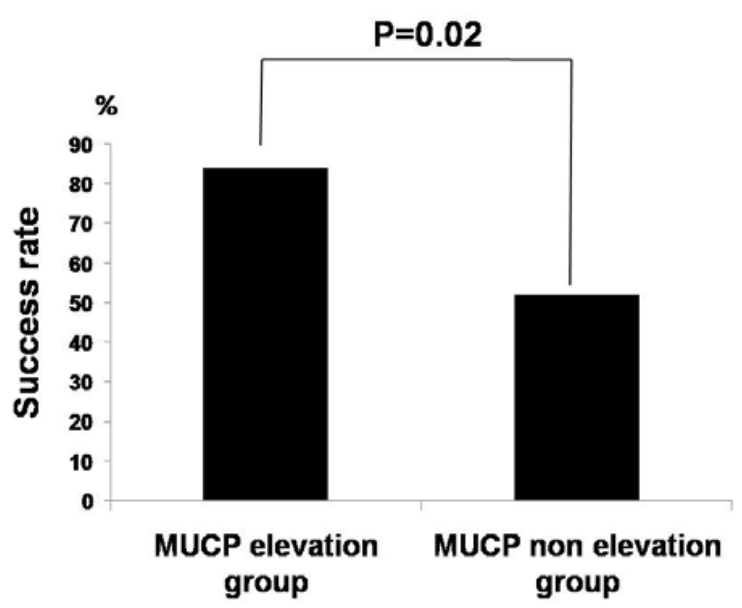

The overall cure rate was significantly higher in the MUCP elevation group compared to the non elevation group (84\% vs. $52 \%)(p=0.02)$.

thought to be based on collagen fibers growing into the tape, which would then fix the tape with mid-urethral tissue rather than tension on the urethra (10).

However, with more experience, many surgeons thought proper tension was needed in the insertion of the tape and that the tension was associated with the surgical outcome. In the case of failed prior mid-urethral sling placement, the tape shortening technique, which shortened the tape to give more tension, had a $60-70 \%$ success rate (5-7). In addition, the Remeex system that allows for tape tension adjustments after surgery was introduced and widely
Figure 2 - The comparison of MUCP change between the cure group and non cure group.

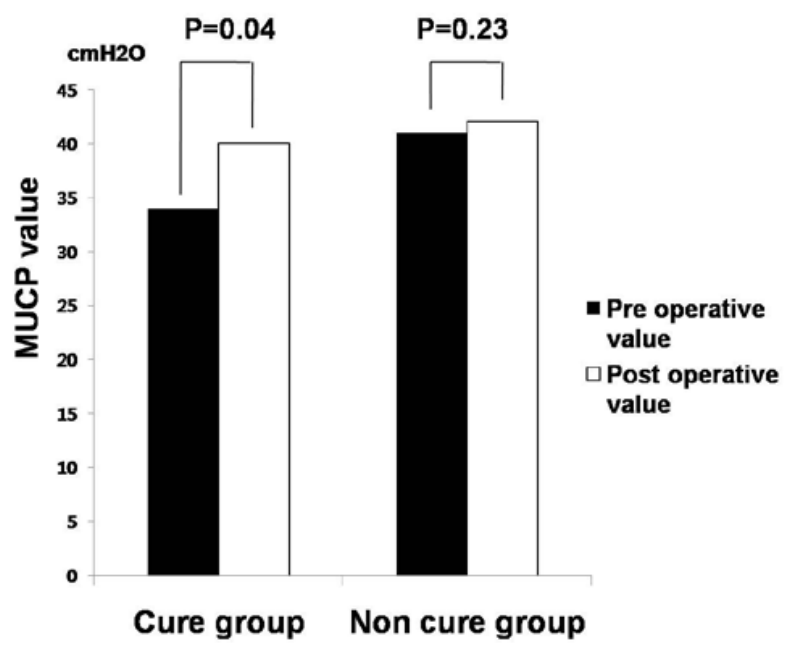

The MUCP increased significantly in the group that was cured $(p=0.04)$; however, there was no difference in the group with a failed procedure $(p=0.23)$.

used (8). The latter two methods were based on the concept that tape tension would produce positive outcomes on continence after sling surgery. Good results using these methods suggest the importance of tension adjustment. Although the tension of the tape has been accepted as an important factor, there has been no study addressing the measurement of the tension and its association with patient outcome.

As there is no standard method of tape tension adjustment, it is usually performed in accordance with the surgeon's experience and feeling. Some surgeons use the surgical scissors or right 
angle clamp between the tape and urethra and others used a bechop clamp to fold the middle part of tape.

Therefore, the purpose of this study was to determine whether tape tension influences surgical outcome and to determine the reference value of proper tension adjustment. We hypothesized that tape tension would influence urethral pressure, which could be measured as the MUCP level in urodynamic testing. The degree of tension was assumed by the change in MUCP before and after placement of the tape using urodynamic studies in the operating room.

The results of this study showed that patients with an elevated MUCP had a higher cure rate compared to patients without an elevated MUCP. An elevated MUCP above $10 \mathrm{cmH}_{2} \mathrm{O}$, after placement of the tape, was associated with a good outcome. Therefore, this factor might be used to help predict patient outcome.

In the non-elevation group, there were patients who showed a decrease in MUCP after tape implantation. So, the mean MUCP was slightly changed.

Similarly, one study reported significant postoperative changes of the MUCP (11). However, another study reported no difference in the MUCP between pre- and post-surgery measurements and no relationship between the outcome of surgery and the UPP (urethral pressure profile) parameters (12). However, in that study, although routine resting UPP had no added value, the postoperative MUCP was also significantly higher in patients with successful outcomes compared to those that had failed surgery (12).

Similarly, the results of this study showed that the mean postoperative MUCP did not differ between patients with successful procedures and failed procedures. The MUCP was decreased in some cases, which made the mean value more similar in the two groups. However, the MUCP was significantly increased in the group with a successful outcome. These findings suggest that the degree of increase in the MUCP rather than the absolute value is the important factor to evaluate during surgery.

The limitations of this study include the following. First, only the UPP and not the URP was measured. The URP may reflect the tension applied to the urethra and further study may show that the value of the URP is an important prognostic factor.
In addition, the cut level of $10 \mathrm{cmH}_{2} \mathrm{O}$ was decided by an analysis that showed a significant difference between the two groups; however, additional studies with more patients are needed to determine the optimal cut-off level.

The patients with MUCP elevation would be expected to have more obstructive symptoms; however, there was no difference in the peak flow rate or residual urine and irritation. One possible explanation is that there was no severe tension that caused residual urine or decreased uroflow in the two groups; even in the group with MUCP elevation. In addition, the number of patients that had urgency or urge incontinence was relatively small.

Although the clinical parameters between the two groups were not significantly different, the prevalence of urinary urgency (32\% compared to $46 \%$ ) was higher in the non-elevation group, and this difference might be clinically relevant.

However, p-value was 0.06, Also, the prevalence of urge incontinence and detrusor overactivity in the urodynamic study were similar between the two groups.

The operator was aware of the baseline MUCP results, and this factor could influence the surgeon's decision during the tape adjustment. However, between the elevation group and the non-elevation group, there is no difference in baseline MUCP. Furthermore, MUCP results before and after placement of the tape were blinded to the operator, and those results did not influence the tape adjustment.

Finally, we performed the operation under spinal anesthesia. The estimated MUCP discrepancy between what was measured during the MUS procedure under spinal anesthesia and the real pressure generated during outcome assessment is unknown. Spinal anesthesia may affect pressure difference during sling surgery. The pressure difference may be a result of the anesthesia utilized. In one study (13), URP was decreased after spinal anesthesia. Similarly, our study showed that the baseline MUCP under no anesthesia is higher than preoperative MUCP under spinal anesthesia. However, the point of our study is that the MUCP difference prior to and after tape insertion is an important prognostic factor, and this is the indirect support needed for proper tension during tape implantation. 
Despite these limitations, this preliminary study shows several implications. First, to our knowledge, there is no study about tape tension influencing sling surgery outcome. Our study showed that tape tension, which was indirectly measured by MUCP elevation, is an important prognostic factor. This study suggests that the intraoperative MUCP measurement should be a useful method for deciding on the appropriate tension adjustment and introduces a method for the quantification of the tension using urodynamic studies.

The feasibility of performing urethral closure pressures pre-and intraoperatively may be questioned. However, intraoperative UDS measurement is not so difficult and it may only take few more minutes. We are convinced of the usefulness of the UDS study because the other known prognostic factors, such as low VLPP, cystocele, mixed incontinence, and other factors are almost uncorrectable. However, MUCP elevation can be measured during surgery and can therefore be adjusted.

In this study, the tape tension was not adjusted when MUCP was not elevated more than $10 \mathrm{~cm}-$ $\mathrm{H}_{2} \mathrm{O}$. It is another topic and further prospective study will be needed to show the effect of tape tension on surgical outcome.

\section{CONCLUSIONS}

The overall cure rate was significantly higher in patients with MUCP elevation compared to patients without MUCP elevation. The change in the MUCP might be a prognostic factor for patient outcome. The intraoperative MUCP measurement was a useful method for deciding on the appropriate tension adjustment.

\section{CONFLICT OF INTEREST}

None declared.
ABBREVIATIONS:
ISD: intrinsic sphincter deficiency
MUCP: maximal urethral closing pressure
MUI: mixed urinary incontinence
PVR: post void residuals
SUI: stress urinary incontinence

TOT: transobturator tape

URP: urethral retro-resistance pressure

UPP: urethral pressure profile

VLPP: valsalva leak point pressure

\section{ACKNOWLEDGEMENT}

This work was supported by Konkuk University in 2011

\section{REFERENCES}

1. Ulmsten U, Henriksson L, Johnson P, Varhos G: An ambulatory surgical procedure under local anesthesia for treatment of female urinary incontinence. Int Urogynecol J Pelvic Floor Dysfunct. 1996; 7: 81-5; discussion 85-6.

2. Kim NS, Bae JH, Lee JG: Long-term follow-up of the tension free vaginal tape (TVT) procedure for treating female stress urinary incontinence. Korean J Urol. 2006; 47: 729-33.

3. Nazemi TM, Yamada B, Govier FE, Kuznetsov DD, Kodama K, Kobashi KC: Minimum 24-month followup of the sling for the treatment of stress urinary incontinence. J Urol. 2008; 179: 596-9.

4. Cetinel B, Demirkesen O, Onal B, Akkus E, Alan C, Can G: Are there any factors predicting the cure and complication rates of tension-free vaginal tape? Int Urogynecol J Pelvic Floor Dysfunct. 2004; 15: 18893.

5. Koh JS, Kim HS, Kim HW, Lee YS, Kim SI, Lee $\mathrm{KS}$, et al.: Comparison of secondary procedures for recurrent stress urinary incontinence after a transobturator tape procedure: shortening of the tape versus tension-free vaginal tape redo. Korean J Urol. 2007; 48: 1149-54.

6. Nam SB, Bae JH, Lee JG: Surgical considerations for recurrent stress urinary incontinence after the midurethral sling procedure: redo midurethral sling and shortening of the tape. Korean J Urol. 2007; 48: 52735.

7. Lo TS, Wang AC, Liang CC, Long CY, Lee SJ: Treatment for unsuccessful tension-free vaginal tape operation by shortening pre-implanted tape. J Urol. 2006; 175: 2196-9; discussion 2199-200.

8. Sousa-Escandón A, Cabrera J, Mantovani F, Moretti M, Ioanidis E, Kondelidis N, et al.: Adjustable suburethral sling (male remeex system) in the treatment of male stress urinary incontinence: a multicentric European study. Eur Urol. 2007; 52: 1473-9. 
9. Haylen BT, de Ridder D, Freeman RM, Swift SE, Berghmans B, Lee J, et al.: An International Urogynecological Association (IUGA)/International Continence Society (ICS) joint report on the terminology for female pelvic floor dysfunction. Neurourol Urodyn. 2010; 29: 4-20.

10. Petros PE, Ulmsten UI: An integral theory and its method for the diagnosis and management of female urinary incontinence. Scand J Urol Nephrol Suppl. 1993; 153: 1-93.
11. Wang AC: An assessment of the early surgical outcome and urodynamic effects of the tension-free vaginal tape (TVT). Int Urogynecol J Pelvic Floor Dysfunct. 2000; 11: 282-4.

12. Wadie BS, El-Hefnawy AS: Urethral pressure measurement in stress incontinence: does it help? Int Urol Nephrol. 2009; 41: 491-5.

13. Duckett RA, Grapsas P, Eaton M, Basu M: The effect of spinal anaesthesia on urethral function. Int Urogynecol J Pelvic Floor Dysfunct. 2008; 19: 257-60.

Submitted for publication:

January 10, 2011

\section{Correspondence address:}

Dr. Hyoung Keun Park

Department of Urology,

College of medicine, Konkuk University

4-12 Hwayang-dong, Gwangjin-gu

Accepted after revision:

April 15, 2011

Seoul, Korea 143-729

Fax: + 02 2030-5319

E-mail:drurol@naver.com 